\title{
Surface and bulk properties of surfactants used in fire-fighting
}

\author{
Christopher Hill, Adam Czajka, Gavin Hazell, \\ Isabelle Grillo, Sarah E. Rogers, \\ Maximilian W.A. Skoda, Nigel Joslin, John Payne \\ and Julian Eastoe
}

\section{Published version information}

Citation: C Hill et al. "Surface and bulk properties of surfactants used in firefighting." Journal of Colloid and Interface Science, vol. 530 (2018): 686-694.

DOI: $\underline{10.1016 / \text { j.jcis.2018.07.023 }}$

(C)2018. This manuscript version is made available under the CC-BY-NC-ND 4.0 Licence.

This version is made available in accordance with publisher policies. Please cite only the published version using the reference above. This is the citation assigned by the publisher at the time of issuing the AAM. Please check the publisher's website for any updates. 
Regular Article

\title{
Surface and bulk properties of surfactants used in fire-fighting
}

\author{
Christopher Hill a , Adam Czajka ${ }^{a}$, Gavin Hazell ${ }^{a}$, Isabelle Grillo ${ }^{b}$, Sarah E. Rogers ${ }^{c}$, \\ Maximilian W.A. Skoda ${ }^{\mathrm{C}}$, Nigel Joslin ${ }^{\mathrm{d}}$, John Payne ${ }^{\mathrm{d}}$, Julian Eastoe ${ }^{\mathrm{a}, *}$
}

a School of Chemistry, University of Bristol, Cantock's Close, Bristol BS8 1TS, UK

${ }^{\mathrm{b}}$ Institut Laue-Langevin, 71 avenue des Martyrs - CS 20156, 30842 Grenoble Cedex 9, France

' ISIS Pulsed Neutron and Muon Source, Science and Technology Facilities Council, Rutherford Appleton Laboratory, Harwell, UK

${ }^{\mathrm{d}}$ Angus Fire Ltd., Station Road, Bentham LA2 7NA, UK

\section{G R A P H I C A L A B S T R A C T}

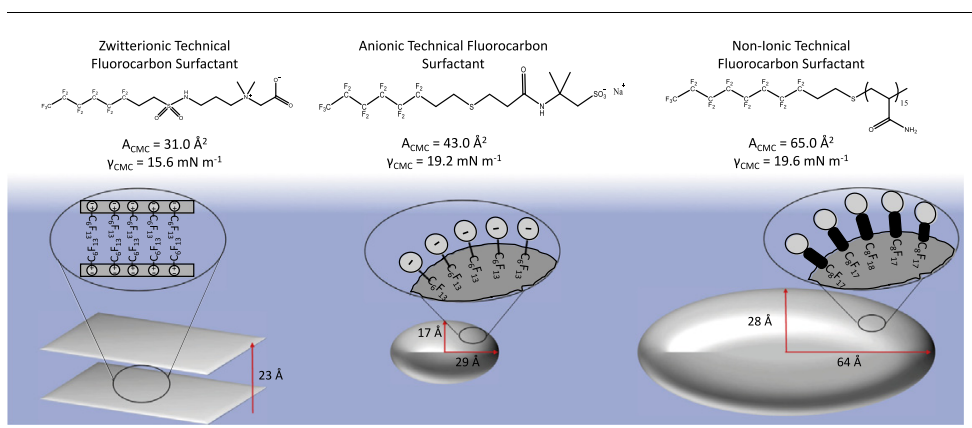

\section{A R T I C L E I N F O}

\section{Article history:}

Received 10 May 2018

Revised 4 July 2018

Accepted 6 July 2018

Available online 7 July 2018

\section{Keywords:}

Fluorocarbon surfactants

Self-assembly

Neutron reflection

Small-angle neutron scattering

Fire-fighting foam formulations

\begin{abstract}
A B S T R A C T
Hypothesis: Reports on the colloidal and interfacial properties of fluorocarbon (FC) surfactants used in fire-fighting foam formulations are rare. This is primarily because these formulations are complex mixtures of different hydrocarbon (HC) and fluorocarbon (FC) surfactants. By developing a greater understanding of the individual properties of these commercial FC surfactants, links can be made between structure and respective surface/ bulk behaviour. Improved understanding of structure property relationships of FC surfactants will therefore facilitate the design of more environmentally responsible surfactant replacements.

Experiments: Surface properties of three partially fluorinated technical grade surfactants were determined using tensiometry and neutron reflection (NR), and compared with a research-grade reference surfactant (sodium perfluorooctanoate (NaPFO)). To investigate the bulk behaviour and self-assembly in solution, small-angle neutron (SANS) scattering was used.

Findings: All FC surfactants in this study generate very low surface tensions $\left(<20 \mathrm{mN} \mathrm{m}^{-1}\right)$ which are comparable, and in some cases, lower than fully-fluorinated surfactant analogues. The complementary techniques (tensiometry and NR) allowed direct comparison to be made with NaPFO in terms of adsorption parameters such as surface excess and area per molecule. Surface tension data for these technical grade FC surfactants were not amenable to reliable interpretation using the Gibbs adsorption equation, however NR provided reliable results. SANS has highlighted how changes in surfactant head group structure can affect bulk properties. This work therefore provides fresh insight into the structure property relationships of some industrially relevant FC surfactants, highlighting properties which are essential for development of more environmentally friendly replacements.
\end{abstract}

() 2018 Elsevier Inc. All rights reserved.

\footnotetext{
* Corresponding author.

E-mail address: julian.eastoe@bristol.ac.uk (J. Eastoe).
} 


\section{Introduction}

Hydrocarbon fuel fires pose a serious threat and as such require a rapid response. Hence, effective and efficient fire-extinguishing agents are needed to prevent re-ignition of these fires. Historically, water has long been used for suppressing fires, however it is ineffective for oily liquid fuel fires [1]. Early advances (1920s-1950s) in fire-fighting found that incorporation of proteinacious materials, such as hydrolysed hoof and horn meal, as well as other natural products, namely saponine or liquorice were beneficial $[1,2]$. The 1960s saw progress mainly in the use of synthetic surfactant formulations, which lead to the development of what are now known as aqueous film forming foams (AFFFs). AFFFs were and still are the most effective formulations for extinguishing fires involving flammable liquid fuels [3]. As with most commercial formulations, AFFFs comprise complex mixtures, incorporating major components such as a solvent (typically a glycol ether), fluorocarbon (FC) (perfluorinated anionic and partially fluorinated amphoteric) surfactants, and hydrocarbon-based surfactants. Table S1 in supporting information shows the composition of a typical AFFF formulation.

Fluorocarbon (FC) surfactants are distinctly different from hydrocarbon (HC) surfactants in various respects. Although the polar headgroups of HC and FC surfactants may be similar, non-polar FC tails have both hydrophobic and oleophobic (oil-repelling) properties, compared to HC surfactants which are considered to be only hydrophobic [4]. Hence, FC surfactants exhibit both hydrophobic and oleophobic characteristics, which in fire-fighting applications, account for their effectivness. In addition to this, FC surfactants generally display greater surface activity compared to HC analogues. Fluorine has a lower polarisability than hydrogen; therefore, the total dispersion interaction is lower for the interaction between fluorinated chains. Hence, FC surfactants are expected to have weaker attractive intermolecular forces than similar HC surfactants. In comparison to those of analogous HC, FC surfactants have larger volume of perfluoroalkyl moieties and larger limiting cross-sectional area [4,5]. As a result, FC surfactants show an enhanced tendency to self-assemble, and collect at the air-water interface to reduce the surface energy. For this reason, incorporation of FC surfactants into AFFFs leads to an increase in spreading coefficient over a hydrocarbon fuel surface, therefore more efficient extinguishment. More information can be found in the following references [6-8].

Although FC surfactants have many useful interfacial properties, and appear in a diverse range of applications, it has been known for many years that they are not environmentally friendly [9-11]. For example, It has been identified that $\mathrm{FC}$ with $\mathrm{C}_{8}-\mathrm{C}_{15}$ chain lengths are hazardous pollutants [12]. These molecules eventually break down to form PFOS (perfluorooctanesulphonate) and PFOA (perfluorooctanoic acid), which are recognised as having negative impacts on the environment and human health due to pronounced persistence, variable degrees of bioaccumulation potential and toxicity $[3,12]$. Although new FC surfactants have been designed which are not bioaccumulative or toxic [13], the strength of the C-F bond hinders biodegradability.

The current understanding underpinning the use of AFFFs for fire-fighting applications is primitive and largely empirically based. As a result of this, few attempts have been made to model or investigate the behaviour of fire-fighting foam formulations from chemical perspectives [14-16]. Therefore, it is important to develop a more fundamental understanding of how the surfactants adsorb and self-assemble, both individually and as mixed systems in mimics of the real formulations ( $\mathrm{F} / \mathrm{F}$ mixtures and $\mathrm{F} / \mathrm{H}$ mixtures).

This study is based on understanding the important structureproperty relationships for individual FC surfactants used in typical fire-fighting foam applications. Studies with techniques such as force tensiometry, neutron reflection (NR) and small-angle neutron scattering (SANS) have allowed the determination of the important properties of typical AFFF FC surfactants from a chemical perspective. As well, this work demonstrates how changing the head group on a FC surfactant (anionic, non-ionic and zwitterionic) feeds through to marked changes to the interfacial properties.

\section{Materials and methods}

\subsection{Materials}

All fluorinated surfactants, apart from sodium perfluorooctanoate (NaPFO), used in this study were provided by commercial suppliers (see below). Dynax ${ }^{\mathrm{TM}}$ DX1030 is a C6 anionic fluorosurfactant (IUPAC Name: 2-methyl-2-(3- $((1 \mathrm{H}, 1 \mathrm{H}, 2 \mathrm{H}, 2 \mathrm{H}-$ perfluoro-1-oct yl) thio) propanamido) propane-1-sulfonate), Dynax ${ }^{\mathrm{TM}} \mathrm{DX} 2200$ is a C8 non-ionic fluorosurfactant and Capstone ${ }^{\mathrm{TM}} 1157$ is a C6 zwitterionic fluorosurfactant (IUPAC Name: $\mathrm{N}$-(carboxymethyl)- $\mathrm{N}$, $\mathrm{N}$-dimethyl-3-(1H,1H,2H,2H-perfluoro-1-octanesulfonamido) propan-1-aminium) Dynax ${ }^{\mathrm{TM}}$ is a trademark of Dynax Corporation and Capstone ${ }^{\mathrm{TM}}$ is a trademark or The Chemours Company. Technical grade surfactants were provided as liquid formulations, acetone was added as a non-solvent to induce precipitation of the solid surfactants for purification. Characterisation and chemical analysis were used to access purity of the precipitated FC surfactants (supporting information), showing the chemical compositions and spectral properties to be consistent with expected molecular structures. NaPFO (CAS 335-95-5) was prepared and purified by the following method. Perfluorooctananoic acid (CAS 335-67-1) of stated purity $\geqslant 99 \%$ was obtained from Fluorochem, coverted into the appropriate metal salt by reaction with the stoichiometric amount of sodium hydroxide, and purified by recrystallisation from a mixture of ethanol and propanol (1:1, vol:vol). Further purification included Soxhlet extraction, with ethyl acetate as solvent, to remove residual inorganic material, and foam fractionation to remove hydrophobic impurities (following method in Ref. [17]). Pyrene (Acros, puriss $\geqslant 99 \%$ ), deuterium oxide (Aldrich, $99.9 \%$ ) were used as received.

\subsection{Methods}

\subsubsection{Surface tension measurements}

Surface tension measurements were carried out on a Krüss K100 force tensiometer using the Wilhemy plate method at $25^{\circ} \mathrm{C}$. Glassware was washed thoroughly with a dilute Decon solution and then with wash cycles of methanol and ultra pure water (Millipore, $18.2 \mathrm{M} \Omega \mathrm{cm}$ ). The cleaning cycles were repeated until the surface tension of water was returned as $72 \pm 0.2 \mathrm{mN} \mathrm{m}^{-1}$.

Stock surfactant solutions were prepared and aliquots were added to deionised water to give desired concentrations. Each concentration measurement was repeated over a period of up to $30 \mathrm{~min}$ to ensure equilibration. Attempts were made to model the data using the Gibbs adsorption isotherm, which relates the surface excess (inversely proportional to the area per molecule) to changes in the surface tension with concentration:

$$
\begin{aligned}
& \Gamma=-\frac{1}{m R T} \frac{d \gamma}{d \ln C} \\
& A_{c m c}=\frac{1}{\Gamma_{c m c} N_{A}}
\end{aligned}
$$

where $\Gamma$ is the surface excess, $\Gamma_{c m c}$ is the surface excess at the critical micelle concentration (CMC), $A_{c m c}$ is the area per molecule at the CMC, $\mathrm{R}$ is the gas constant, $\mathrm{T}$ is temperature, $\gamma$ is surface tension, 
$C$ is concentration and $N_{A}$ is the Avogadro number. The prefactor $m$ is dependent on the surfactant type and structure, as well as the presence of extra electrolyte in the aqueous phase [18]. For nonionic and zwitterionic surfactants, a value of 1 for the pre-factor has been confirmed $[19,20]$, and for $1: 1$ ionic surfactants, in the absence of extra electrolyte, $\mathrm{m}=2$ has been confirmed [18]. Recently, it has been shown that traces of multivalent ionic impurities can lead to changes in this prefactor, therefore leading to problems employing the Gibbs adsorption isotherm [21]. In this study, a prefactor of 1 has been used for the non-ionic and zwitterionic surfactants, and a prefactor of 2 has been used for the anionic surfactants, following literature [18-20].

\subsubsection{Fluorescence}

Fluorescence measurements were carried out as described in Ref. [22] in quartz cuvettes at $25^{\circ} \mathrm{C}$ on a Cary Eclipse (Varian) Fluorescence spectrometer. Pyrene was used as an hydrophobic fluorescent probe for determining CMCs of the studied surfactants. A fixed concentration of pyrene $\left(1.0 \times 10^{-6} \mathrm{M}\right)$ was added to sample vials from a known stock prepared in acetone. The acetone was allowed to evaporate off in air, leaving behind a known mass of the involatile pyrene, before the aqueous surfactant solutions were added over the concentration range of interest. Fluorescence emission spectra were collected after excitation at $\lambda=337 \mathrm{~nm}$, with a slit width of $5 \mathrm{~nm}$ for excitation and emission. Each measurement was repeated three times.

\subsubsection{Neutron reflection}

Neutron reflection (NR) measurements were conducted using the INTER beam-line on Target Station 2 at the ISIS facility (Rutherford Appleton Laboratory, Didcot, UK) [23]. Measurements were taken using a single point detector and fixed grazing incidence angles $\left(0.8^{\circ}\right.$ and $\left.2.3^{\circ}\right)$. The absolute reflectivity was calibrated with respect to the direct beam and the reflectivity from a clean $\mathrm{D}_{2} \mathrm{O}$ surface. The NR experiments were carried out in two contrasts, pure $\mathrm{D}_{2} \mathrm{O}$ and air contrast matched water (ACMW; $8 \mathrm{~mol} \% \mathrm{D}_{2} \mathrm{O}$ in $\mathrm{H}_{2} \mathrm{O}$ with a scattering length density (SLD) of 0 ). A pipette was used on each sample to suck off any air bubbles and also to remove the inital surface layer in case any hydrophobic impurities were present. The data were fit using MOTOFIT, written for IGOR Pro [24].

Only the relevant theory is described here, but for a more indepth account, the reader the referred to the following references $[25,26]$. The specular reflection of neutrons is measured as a function of the scattering vector, $\mathrm{Q}$, as given by:

$Q=\frac{4 \pi \sin \theta}{\lambda}$

where $\lambda$ is neutron wavelength, and $\theta$ is the angle of half the reflection. The experimental reflectivity is related to the square of the Fourier transform of the SLD, $\rho(z)$, normal to the surface. For neutrons, $\rho(z)=\Sigma_{i} n_{i}(z) \cdot b_{i}$, where $n_{i}$ and $b_{i}$ are the number density and scattering length of the ith component and $z$ is the direction perpendicular to the surface [26]. For surfactant films of this kind, the measured reflectivity curve can be modeled in terms of a single, uniform layer to fit for thickness, $\tau$, and a scattering length density, $\rho$ [25]. These values are related to the surface coverage, $\Gamma$, and area per molecule, $A$, in the following way:

$A=\frac{\Sigma b_{i}}{\rho \tau}=\frac{1}{\Gamma N_{A}}$

where $\Sigma b_{i}$ is the sum of neutron scattering lengths of nuclei over the surfactant molecule, $\Gamma$ is the surface coverage and $N_{A}$ is the Avogadro number.

\subsubsection{Small-angle neutron scattering}

SANS measurements were performed on SANS 2D at the ISIS facility (Rutherford Appleton Laboratory, Didcot, UK) and D33 at the Institut Laue-Langevin (ILL, Grenoble, France). On SANS 2D, a simultaneous $Q$-range of $0.004-0.6 \AA^{-1}$ was achieved with a neutron wavelength range of $1.75<\lambda<15.5 \AA$ and a source-sampledetector distance $\mathrm{L} 1=\mathrm{L} 2=4 \mathrm{~m}$. The $\mathrm{D} 33$ instrument used neutrons with a wavelength of $\lambda=6 \AA$ and two sample-detector positions ( 2 and $7.5 \mathrm{~m}$ ) providing an accessible $Q$ range of $0.005-$ $0.2 \AA^{-1}$. All samples were made in $\mathrm{D}_{2} \mathrm{O}$, using $2 \mathrm{~mm}$ path length rectangular quartz cells at a temperature of $25^{\circ} \mathrm{C}$. Raw SANS data were reduced by subtracting the scattering of the empty cell and the $\mathrm{D}_{2} \mathrm{O}$ background and normalised to an appropriate standard using the instrument-specific software. SANS data were fit using the analysis package SasView.

In a SANS experiment, the intensity $(I)$ of scattered neutrons is measured as a function of momentum transfer $(Q)$, see Eq. (3). For monodispersed homogeneous scatterers of volume $V_{p}$, number density $N_{p}$ and coherent scattering length density $\rho_{p}$, dispersed in a solvent of scattering length density $\rho_{s}$, the normalised SANS intensity $\mathrm{I}(\mathrm{Q})\left(\mathrm{cm}^{-1}\right)$ is:

$I(Q)=\phi V_{P}\left(\rho_{p}-\rho_{s}\right)^{2} P(Q) S(Q)$

where $\phi=(N / V) V_{p}$. The first three terms in Eq. (5) are independent of $Q$ and account for the absolute intensity of scattering. The last two terms in the equation are $Q$-dependent functions. $P(Q)$ is the particle form factor, which describes intra-particle information such as size and shape. $S(Q)$ is the structure factor, which describes the scattering due to inter-particle correlations.

\section{Results and discussion}

\subsection{Equilibrium surface tensions and Critical Micelle Concentrations (CMCs)}

Important parameters to determine for surfactants are how effective they are at reducing aqueous surface tension and critical micelle concentration (CMC). In fire-fighting applications, properties such as foamability, foam stability and spreading are linked to surface tension reductions. For example, it is often believed that employing surfactants at their CMCs gives the best foam performance [27].

Results from characterisation of the pure research-grade surfactant, sodium perfluorooctanoate (NaPFO) were compared to literature data $[28,29]$.

\subsubsection{Properties of NaPFO}

An equilibrium $\gamma$ vs. In (concentration) plot NaPFO is shown in Fig. 2 having a clear break point at the CMC, with no minimum or shoulder, which would be indicative of hydrophobic impurities. The CMC was determined by taking the second derivative of the $\gamma$ vs. $\ln$ (concentration) plots, and then applying a Gaussian distribution function, where the minimum was taken to be the value of the CMC. This method is described in supporting information [30]. Quartic functions were then fit through the pre-CMC surface tension data, to generate local tangents, then the Gibbs adsorption isotherm was used to estimate the surface excess $\left(\Gamma_{c m c}\right)$ and the area per molecule $\left(\mathrm{A}_{c m c}\right)$ at the CMC using Eq. (1), data shown in Table 1.

Similar results for NaPFO have been reported [28,29] for both $\Gamma_{c m c}$ and $\mathrm{A}_{c m c}$ and are comparable to the results achieved here. This shows that the standard tensiometric method used here is amenable for analysis of pure research-grade fluorocarbon surfactants of this kind. 
<smiles>O=C([18O])CCCCC(F)C(F)(F)F</smiles>

(a) Sodium Perfluorooctanoate<smiles>CC(CSCC[Te]CCPCCPCCPC(F)(F)F)C(C)(C)C</smiles>

(c) Dynax ${ }^{\mathrm{TM}}$ DX2200

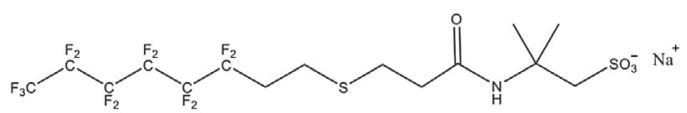

(b) Dynax ${ }^{\mathrm{TM}} \mathrm{DX} 1030$

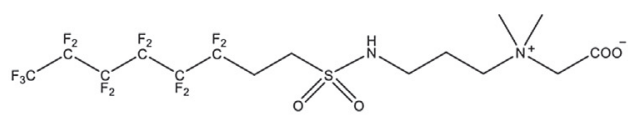

(d) Capstone $^{\mathrm{TM}} 1157$

Fig. 1. Surfactants Used In This Work.
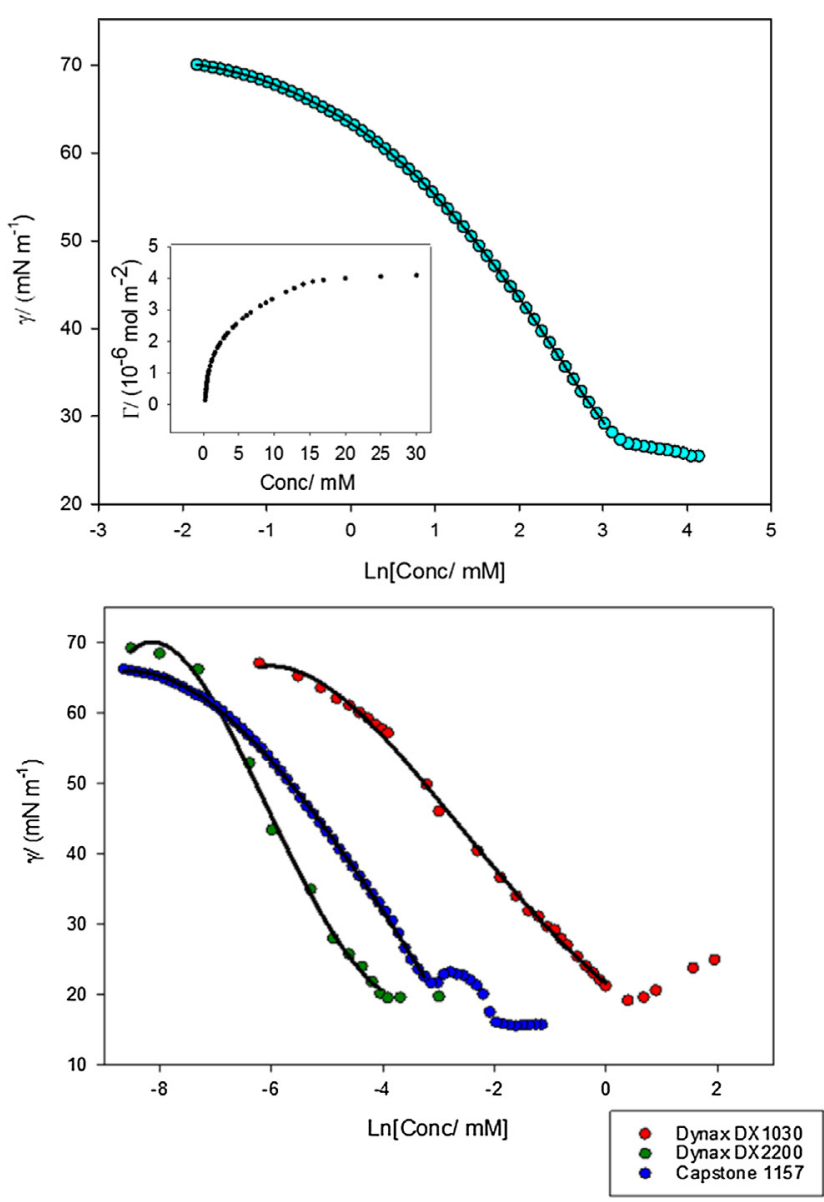

Fig. 2. Above: Surface Tension vs. In(Concentration) for NaPFO, inset shows the adsorption isotherm of NaPFO. Below: Surface Tension vs. $\ln$ (Concentration) for three technical grade Surfactants. $\mathrm{T}=25^{\circ} \mathrm{C}$. Line fitted to pre-CMC data is a quartic function.

\subsubsection{Technical grade surfactants}

Equilibrium $\gamma$ vs. $\ln$ (concentration) plots for the three firefighting surfactants are shown in Fig. 2. Dynax ${ }^{\mathrm{TM}}$ DX2200 (nonionic) and Capstone ${ }^{\mathrm{TM}} 1157$ (zwitterionic) show clear break points at their respective $\mathrm{CMCs}$, therefore relatively accurate $\mathrm{CMC}$ values can be determined. On the other hand, Dynax ${ }^{\mathrm{TM}}$ DX1030 (anionic) shows a minimum in the curve, followed by an increase in surface tension, which is indicative of hydrophobic impurities, as first recognised by Mysels et al. [31,32]. CMCs were determined using the same method as previously mentioned, this is also true for calculation of both the surface excess $\left(\Gamma_{c m c}\right)$ and the area per molecule $\left(\mathrm{A}_{c m c}\right)$, data shown in Table $\mathrm{S} 8$ in the supporting information. Although it has been possible to attain CMCs, fluorescence probe measurements have been used to provide additional CMC values, matching well with the surface tension results (Table S9, supporting information).

Due to the nature of the surfactants, and the irregular forms of these surface tension isotherms (Fig. S5), it is clear that problems will arise when attempting to use the Gibbs adsorption isotherm as described above. This is reflected in the erratic and sometimes unphysical data shown in Table S8 (supporting information), and obtained results should therefore be considered with caution. Taking the surface tension curve of Dynax ${ }^{\mathrm{TM}}$ DX1030 as an example, it can be seen that the line fitted through the data is straight, suggesting from the Gibbs adsorption isotherm that $\Gamma$ is essentially constant. However, this clearly cannot be the case, as the surface tension is reducing. It might be expected that NaPFO and anionic Dynax $^{\mathrm{TM}}$ DX1030 should achieve similar values for both surface excess and $\mathrm{A}_{c m c}$ due to their similar tail structures and head groups, however, this is also not seen in the results. Dynax ${ }^{\mathrm{TM}}$ DX1030 was found to have $A_{c m c}$ two times larger and a surface excess lower than SPFO, suggesting that use of the Gibbs adsoprtion isotherm for these technical grade surfactants should be treated with caution. In addition, anionic Dynax ${ }^{\mathrm{TM}}$ DX1030 and zwitterionic Capstone $^{\mathrm{TM}} 1157$ are similar in both molecular size and level of fluorination (see Fig. 1), therefore similar values for $A_{c m c}$ and $\Gamma_{c m c}$ would be expected. The anionic charge on Dynax ${ }^{\mathrm{TM}}$ DX1030 would be expected to cause an increase in $A_{c m c}$, and thus a decrease in $\Gamma_{c m c}$ due to head group repulsion, however the difference observed between Capstone ${ }^{\mathrm{TM}} 1157$ and Dynax ${ }^{\mathrm{TM}} \mathrm{DX} 1030$ does not fit in with this. Dynax ${ }^{\mathrm{TM}} \mathrm{DX} 2200$ is non-ionic with repeating acrylamide units: a similar molecule was studied by Dupont et al. [33]. Their work was carried out with a tris(hydroxymethyl) acrylamidomethane (THAM)-derived telomer bearing a perfluorohexyl hydrophobic chain. In that case, $\Gamma_{c m c}=2.68 \times 10^{-6} \mathrm{~mol} \mathrm{~m}^{-2}$ and a $A_{c m c}$ of 62 $\pm 2 \AA^{2}$, was observed.

Table 1

Results from surface tension measurements of NaPFO from this study compared to literature [28,29].

\begin{tabular}{|c|c|c|c|c|}
\hline Surfactant & $\gamma_{C M C} /\left(\mathrm{mN} \mathrm{m}^{-1}\right)$ & $\mathrm{CMC} /(\mathrm{mM})$ & $\Gamma_{c m c} /\left(10^{-6} \mathrm{~mol} \mathrm{~m}^{-2}\right)$ & $\mathrm{A}_{c m c} /\left(\AA^{2}\right)$ \\
\hline NaPFO (This Study) & $22.6 \pm 0.1$ & $25.00 \pm 0.05$ & $4.07 \pm 0.20$ & $40 \pm 2$ \\
\hline NaPFO (Literature) & 24.6 & 30.0 & 4.0 & 42 \\
\hline
\end{tabular}


Therefore, it seems that using tensiometric techniques and analysis with the Gibbs adsorption isotherm is not appropriate for accurate detail on the interfacial properties of these technical grade FC surfactants, resulting in unphysical values for both $A_{c m c}$ and $\Gamma_{c m c}$. As a way to circumvent this issue and to determine these important parameters of interest, Neutron Reflection has been employed.

\subsection{Neutron reflection}

Using NR with a fluorocarbon (FC) chain surfactants on air contrast match water (ACMW, i.e. $\rho_{A C M W}=0 \AA^{-2}$ ), the surface coverages can be determined directly from analyses of the reflectivity profiles $R(Q)$ [33,34]. Data were modelled in terms of a single uniform layer to fit for monolayer thicknesses, $\tau$, and scattering length densities $\rho$. Molecular areas and surface excesses were then calculated using the modelled parameters via Eq. (4). The reflectivity curves can be found in the supporting information.

\subsubsection{NaPFO}

As for tensiometry, NaPFO was initially characterised to validate the method and ensuing analyses. The parameters from analysis of the NaPFO R(Q) data can be found in Table 2 and the $\Gamma$ vs. concentration plot calculated using Eq. (4) can be seen Fig. S9 in the supporting information. Here, similar results have been reported when comparing to fitted parameters found in the literature [28,29,35].

Comparisons can also be made between NR and surface tension data in terms of both $\Gamma_{c m c}$ and $\mathrm{A}_{c m c}$. A high degree of agreement should be noted between these two complementary techniques: $\Gamma_{c m c}=4.07 \pm 0.20 \times 10^{-6} \mathrm{~mol} \mathrm{~m}^{-2}(\mathrm{ST})$ and $4.05 \pm 0.10 \times 10^{-6}$ $\mathrm{mol} \mathrm{m} \mathrm{m}^{-2}$ (NR), $\mathrm{A}_{c m c}=40 \pm 2 \AA^{2}$ (ST) and $41 \pm 2 \AA^{2}$ (NR). These results therefore show how using both standard tensiometric methods in conjunction with NR can provide directly comparable results, and are amenable for analysis of research-grade FC surfactants.

\subsubsection{Technical grade surfactants}

It was previously shown that using common tensiometric techniques did not allow accurate analysis of parameters such as $\Gamma_{c m c}$ and $\mathrm{A}_{c m c}$ for the technical grade FC surfactants. Therefore, the basis of this section is to determine whether these important surfactant parameters can be obtained using NR. All surfactants studied in this section have been subject to the same analysis as previously in Section 3.2.1. Example NR curves are shown in Fig. 3 (see supporting information for more data), Table 2 lists fitted values from analyses. The full set of parameters used to fit these data can be found in the supporting information. Comparing the results for the technical grade surfactants with the NaPFO, it can be seen that, as might be expected owing to the similarities in chemical structure (Fig. 1), Dynax ${ }^{\mathrm{TM}}$ DX1030 and NaPFO have similar values for both $\mathrm{A}_{c m c}$ and $\Gamma_{c m c}$. Different thicknesses are expected due to Dynax $^{\mathrm{TM}}$ DX1030 having the additional $\mathrm{CH}_{2}$ groups. Dynax ${ }^{\mathrm{TM}}$ DX1030 and Capstone ${ }^{\mathrm{TM}} 1157$ have a very similar overall molecular size and tail structure, therefore as expected comparable values are observed for fitted layer thickness $(\tau), 26.5 \pm 1.0 \AA$ and $23.0 \pm 0.5$ $\AA$ respectively. Although they are similar in size, differences are observed when considering both $\mathrm{A}_{c m c}$ and $\Gamma_{c m c}$, because Dynax ${ }^{\mathrm{TM}}$ DX1030 is anionic and Capstone ${ }^{\mathrm{TM}} 1157$ zwitterionic. Dynax ${ }^{\mathrm{TM}}$ DX1030 has a larger area per molecule and thus lower surface excess to Capstone $\mathrm{T}^{\mathrm{TM}}$ 1157, due to charge repulsion between head groups [36]. Comparing ST and NR for Capstone ${ }^{\mathrm{TM}}$ 1157, there are similarities between the values for both $\Gamma_{c m c}$ and $A_{c m c}: \Gamma_{c m c}=$ $5.80 \pm 0.1 \times 10^{-6} \mathrm{~mol} \mathrm{~m}^{-2}(\mathrm{ST})$ and $5.22 \pm 0.10 \times 10^{-6} \mathrm{~mol} \mathrm{~m}^{-2}$ (NR), $A_{c m c}=28 \pm 1 \AA^{2}(\mathrm{ST})$ and $31 \pm 1 \AA^{2}$ (NR). Capstone ${ }^{\mathrm{TM}} 1157$ was found to the most amenable to tensiometric measurements and analysis, see supporting information. Another important comparison to make is between the cross-sectional area of a single fluorocarbon chain, which is approximately $28 \AA^{2}$ [37-39]. Well packed fluorocarbon surfactant monolayers generate a low limiting value of $\gamma_{c m c}$ of $15 \mathrm{mN} \mathrm{m}^{-1}$ [5]. This value represents the physical limit at which these surfactant molecules can pack at an
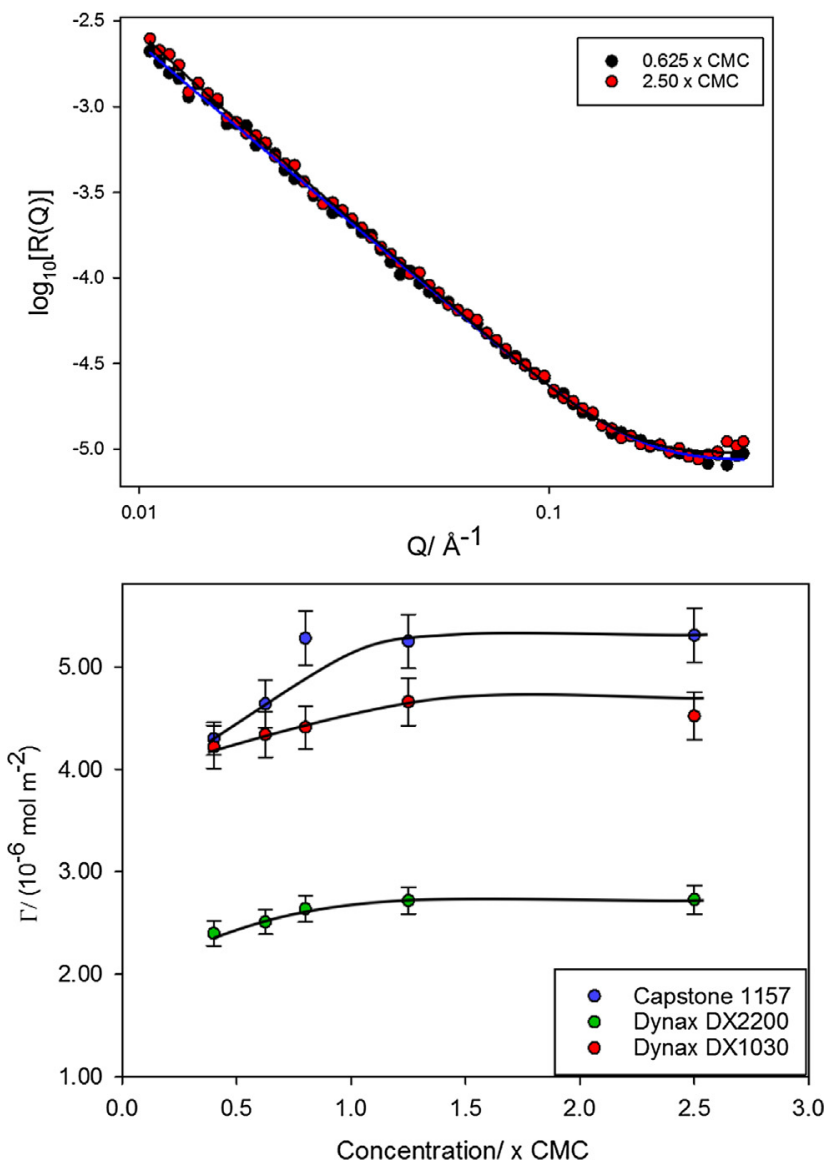

Fig. 3. Above: Capstone ${ }^{\mathrm{TM}} 1157$ neutron reflection profiles to show difference in reflection intensity. Fitted functions shown as lines. Below: Surface excesses obtained by analyses of neutron reflection data. Lines are a guide to the eye. Critical micelle concentrations have been taken as $0.23 \mathrm{mM}, 1.37 \mathrm{mM}$ and $0.02 \mathrm{mM}$ for Capstone ${ }^{\mathrm{TM}} 1157$, Dynax ${ }^{\mathrm{TM}} 1030$ and Dynax ${ }^{\mathrm{TM}} \mathrm{DX} 2200$ respectively. $\mathrm{T}=25^{\circ} \mathrm{C}$.

Table 2

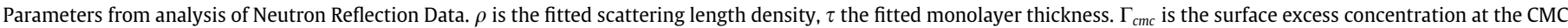
and $\mathrm{A}_{c m c}$ is the area per molecule at the CMC (Eq. (4)). Additional model parameters can be found in Supporting information Table S10.

\begin{tabular}{|c|c|c|c|c|}
\hline Surfactant & $\rho /\left(10^{-6} \AA^{-2}\right)$ & $\tau /(\AA)$ & $\Gamma_{c m c} /\left(10^{-6} \mathrm{~mol} \mathrm{~m}^{-2}\right)$ & $\mathrm{A}_{c m c} /\left(\AA^{2}\right)$ \\
\hline NaPFO (This Study) & 2.50 & $15.0 \pm 0.5$ & $4.05 \pm 0.10$ & $41.0 \pm 2.0$ \\
\hline NaPFO (Literature) & 1.80 & $18.0 \pm 0.5$ & 4.00 & 42 \\
\hline Dynax $^{\mathrm{TM}}$ DX1030 & 1.35 & $26.5 \pm 1.0$ & $3.95 \pm 0.20$ & $42.5 \pm 2.0$ \\
\hline Dynax $^{\mathrm{TM}}$ DX2200 & 2.55 & $25.0 \pm 2.0$ & $2.50 \pm 0.10$ & $65.0 \pm 0.5$ \\
\hline Capstone $^{\mathrm{TM}} 1157$ & 2.00 & $23.0 \pm 0.5$ & $5.22 \pm 0.10$ & $31.0 \pm 1.0$ \\
\hline
\end{tabular}


air-water interface, therefore leading to the lowest achievable value of $\gamma_{\mathrm{cmc}}$. With this in mind, it is interesting to observe the easy to follow trend between $\mathrm{A}_{c m c}$ and limiting value of $\gamma_{c m c}$ between the technical surfactants in this study. The molecule with the largest $\mathrm{A}_{c m c}\left(\right.$ Dynax $^{\mathrm{TM}} \mathrm{DX} 2200$ ) has a $\gamma_{c m c}$ of $\sim 20 \mathrm{mN} \mathrm{m}^{-1}$ (Table S8, supporting information). Capstone $\mathrm{T}^{\mathrm{TM}} 1157$ has the lowest $\mathrm{A}_{c m c}$ of the three technical surfactants (comparable to that of a single fluorocarbon chain), explaining the low observed $\gamma_{c m c}$ of $\sim 16 \mathrm{mN} \mathrm{m}^{-1}$ (Table S8, supporting information).

Due to its larger relative size compared to the two other surfactants, it is expected that Dynax ${ }^{\mathrm{TM}}$ DX2200 will have a smaller surface excess and a larger area per molecule, as reflected in the findings. As previously mentioned, properties of a non-ionic surfactant of similar structure to Dynax ${ }^{\mathrm{TM}}$ DX2200 have been reported in the literature [33]. Parameters derived from analysis of NR data provided comparable results for both $\Gamma_{c m c}$ and $\mathrm{A}_{c m c}$, $\Gamma_{c m c}=2.50 \pm 0.10 \mathrm{~mol} \times 10^{-6} \mathrm{~m}^{-2}$ and $65.0 \pm 0.5 \AA^{2}$ (Dynax DX2200), $\Gamma_{c m c}=2.46 \times 10^{-6} \mathrm{~mol} \mathrm{~m}^{-2}$ and $67 \AA^{2}$ (THAM)-derived telomer).

Neutron reflectivity provides significant information on how these fire-fighting surfactants adsorb and pack at the air-water interface. Although this study has reported results on these FC surfactants as single surfactant systems, it provides a means for fur-

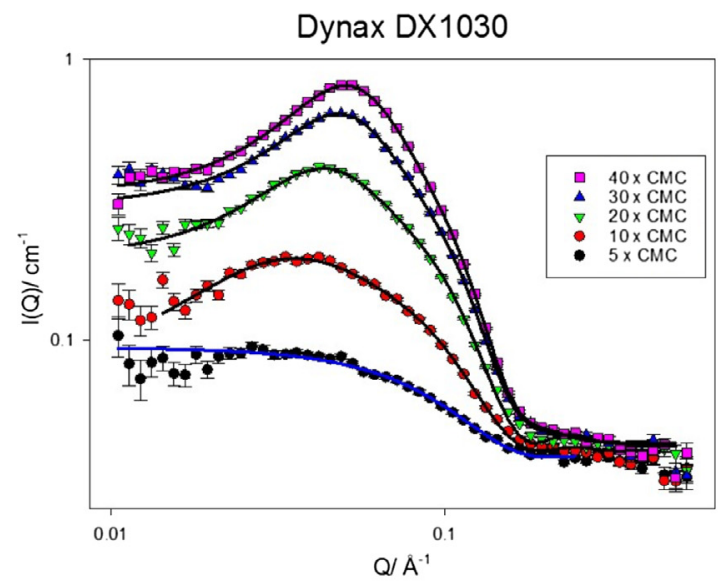

(a) Dynax ${ }^{\mathrm{TM}}$ DX1030 ther investigations into more complex mixed systems, involving F Carbon/ F-Carbon and F-Carbon/ H-Carbon mixtures, as found in some commercial formulations. Overall, it has been shown that NR can be used to achieve consistent and reliable results for studying both research-grade and technical-grade FC surfactants. For the pure NaPFO, the data from both NR and tensiometric techniques were directly comparable to literature data. In addition to this, the three technical grade FC surfactants followed expected trends in terms of $\tau, \Gamma_{c m c}$ and $\mathrm{A}_{c m c}$, unlike the unexpected and erratic trends found using tensiometry.

\subsection{Small-Angle Neutron Scattering (SANS)}

SANS has been employed to investigate self-assembly of the FCsurfactants over multiple concentrations above their respective $\mathrm{CMCs}$ (Table $\mathrm{S} 8$ in supporting information) at $25^{\circ} \mathrm{C}$ in $\mathrm{D}_{2} \mathrm{O}$. The scattering profiles for all three surfactants are shown in Fig. 4. The anionic surfactant, Dynax ${ }^{\mathrm{TM}}$ DX1030 (4a), and the non-ionic surfactant, Dynax ${ }^{\mathrm{TM}}$ DX2200 (4b), are well described by a form factor for oblate spheroids. Parameters for the oblate spheroid form factor are: equatorial radius $\left(R_{e q} / \AA\right)$, polar radius $\left(R_{\text {pol }} / \AA\right)$, aspect ratio $\left(X=R_{e q} / R_{p o l}\right)$ and charge $(Z)$ for the anionic surfactant. The zwitterionic surfactant, Capstone 1157, displays scattering to

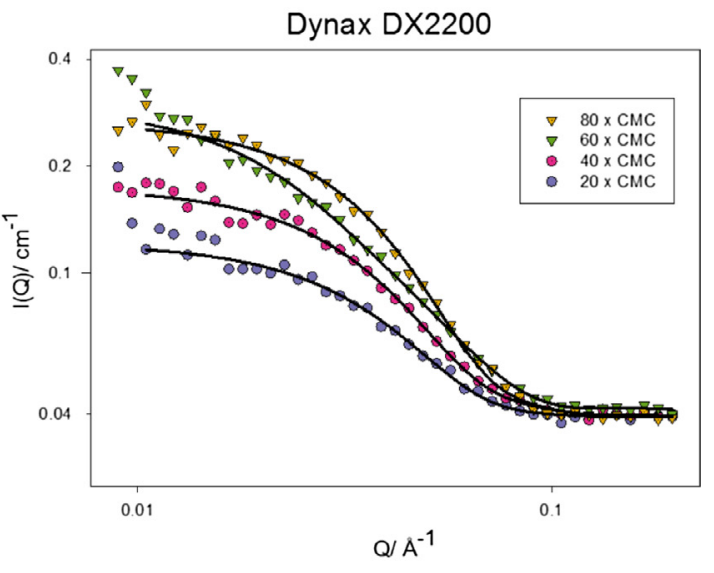

(b) Dynax ${ }^{\mathrm{TM}}$ DX2200

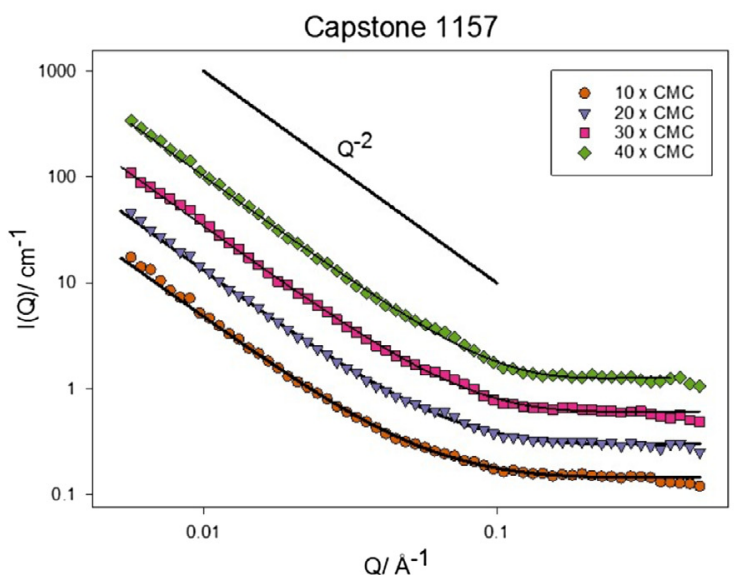

(c) Capstone $\mathrm{CM}^{\mathrm{TM}}$ 1157. Data have been offset by multiplied by 4,16 and 36 respectively for clarity

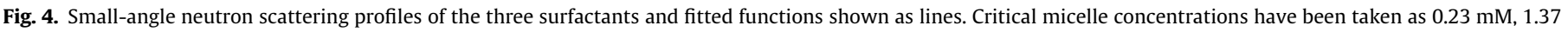
$\mathrm{mM}$ and $0.02 \mathrm{mM}$ for Capstone ${ }^{\mathrm{TM}} 1157$, Dynax ${ }^{\mathrm{TM}} 1030$ and Dynax ${ }^{\mathrm{TM}} \mathrm{DX} 2200$ respectively. $\mathrm{T}=25^{\circ} \mathrm{C}$. 
Table 3

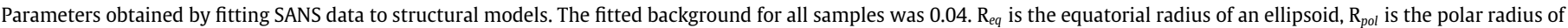

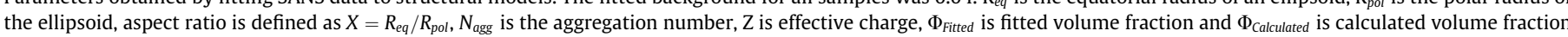

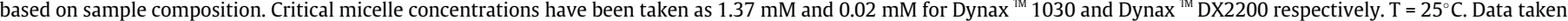
on SANS2D.

\begin{tabular}{|c|c|c|c|c|c|c|c|c|c|}
\hline Surfactant & Concentration (x CMC) & Model & $\mathrm{R}_{e q} /(\AA)( \pm 0.1)$ & $\mathrm{R}_{\text {pol }} /(\AA)( \pm 0.1)$ & Aspect ratio & $N_{\text {agg }}$ & $Z( \pm 1)$ & $\Phi_{\text {Fitted }}$ & $\Phi_{\text {Calculated }}$ \\
\hline \multirow[t]{5}{*}{ Dynax $^{\mathrm{TM}}$ DX1030 } & 5 & Sphere & 22.0 & 22.0 & & 54 & & 0.003 & 0.004 \\
\hline & 10 & Ellipsoid & 29.0 & 17.0 & 1.7 & 97 & 10 & 0.005 & 0.008 \\
\hline & 20 & Ellipsoid & 28.5 & 16.7 & 1.7 & 92 & 7 & 0.011 & 0.016 \\
\hline & 30 & Ellipsoid & 29.0 & 17.0 & 1.7 & 97 & 7 & 0.021 & 0.025 \\
\hline & 40 & Ellipsoid & 30.0 & 17.7 & 1.7 & 108 & 8 & 0.029 & 0.033 \\
\hline \multirow[t]{4}{*}{ Dynax $^{\mathrm{TM}}$ DX2200 } & 20 & Ellipsoid & 61.0 & 31.0 & 2.0 & 212 & & 0.0003 & 0.0006 \\
\hline & 40 & Ellipsoid & 64.0 & 28.0 & 2.3 & 311 & & 0.0009 & 0.0012 \\
\hline & 60 & Ellipsoid & 70.0 & 22.0 & 3.2 & 292 & & 0.0014 & 0.0018 \\
\hline & 80 & Ellipsoid & 63.0 & 31.0 & 2.0 & 333 & & 0.0023 & 0.0025 \\
\hline
\end{tabular}

Table 4

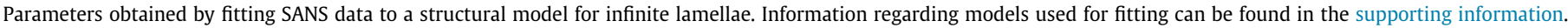
Critical micelle concentrations has been taken as $0.23 \mathrm{mM}$. T $=25^{\circ} \mathrm{C}$. Data taken on SANS2D.

\begin{tabular}{|c|c|c|c|c|}
\hline Surfactant & Concentration $(\times \mathrm{CMC})$ & Background & Model & Thickness $(\AA)( \pm 1)$ \\
\hline \multirow[t]{4}{*}{ Capstone $^{\mathrm{TM}} 1157$} & 10 & 0.07 & Lamellar & 22 \\
\hline & 20 & 0.08 & Lamellar & 23 \\
\hline & 30 & 0.07 & Lamellar & 26 \\
\hline & 40 & 0.07 & Lamellar & 27 \\
\hline
\end{tabular}

much lower values of $Q$ compared to the two other surfactants and these SANS curves have been fitted with a lamellar form factor as infinite sheets of thickness $(\mathrm{T} / \AA)$. The micelle dimensions along with parameters such as background and volume fraction are given in Table 3 and 4.

At low concentration $(5 \times \mathrm{CMC})$, Dynax ${ }^{\mathrm{TM}} \mathrm{DX} 1030$ aggregates appear to be spherical in shape. Whereas, ellipsoidal aggregates are observed at $10 \times \mathrm{CMC}$ and above. The aspect ratio of the aggregates above $10 \times \mathrm{CMC}$ remain relatively constant $(X=1.71 \pm 0.01)$, suggesting that the shape of the micelles is not changing at these higher concentrations (Table 3 ). Another observation is that the structure factor $S(Q)$ peak for the anionic surfactant Dynax ${ }^{\mathrm{TM}}$ DX1030 which occurs at $Q_{\max }$ shifts to higher $Q$ as the concentration is increased. At low concentrations, the $S(Q)$ peak is difficult to discern due to weaker interactions. The $Q_{\max }$ peak provides a rough guide on the average distance between the micelles. This shift to higher $Q$ shows that there is a decrease in the average distance between the micelles with concentration. Similar results have been observed in studies of both anionic hydrocarbon and fluorocarbon surfactants $[40,41]$.

The zwitterionic betaine, Capstone ${ }^{\mathrm{TM}} 1157$ has been studied at four different concentrations above its CMC. The solutions at these concentrations are quite viscous, reminiscent of viscoelastic systems, suggesting the formation of large and/or entangled aggregates. Amphoteric betaines are known to exhibit viscoelastic behaviour in solution, and work by Kumar et al. [42] showed by SANS/ TEM how a C22-amphoteric betaine generates worm-like micelles thus resulting in the observed rheological properties.

In the low- $Q$ region, the scattering scales as $I(Q) \approx Q^{-D}$, where $D$ is a characteristic dimensionality of the dispersed colloids and the gradient of a log-log plot will be $-D$ [43]. In the case of non-interacting spheres, D should be zero in the low- $\mathrm{Q}$ region, for cylinders, $D=1$, and for disks/ lamellar structure $D=2$. Capstone 1157 generates scattering with a clear $Q^{-2}$ dependency, as shown in Fig. 4c, and therefore can be attributed to large lamellar structures across the concentration range studied here. Fitting the data to a lamellar model provides information only on the thickness of the layers. The average fitted thickness over all concen- trations is $\sim 25 \AA$, this value being roughly commensurate with the tip-to-toe length of two of the tail groups for this surfactant.

Considering the molecular structure of non-ionic Dynax ${ }^{\mathrm{TM}}$ DX2200, with a fluorinated tail group and repeating acrylamide unit head group, a complex core-shell model fit was investigated. The model was set up to account for a fluorinated tail micelle core ( $\rho \sim 3 \times 10^{-6} \AA^{-2}$ ) surrounded by a shell of acrylamide unit headgroups $\left(\rho \sim 1 \times 10^{-6} \AA^{-2}\right)$ contrasted against a $\mathrm{D}_{2} \mathrm{O}$ continuum $\left(\rho \sim 6 \times 10^{-6} \AA^{-2}\right)$. Attempts to determine a model fit for the system using this approach provided unphysical values for parameters, suggesting that the internal structure of these micelles cannot be resolved by SANS in this case. Several reasons may explain this: (1) Perhaps because there is only effectively a relatively small contrast step across the interface; (2) Blurring of the contrast step at the headgroup $/ \mathrm{D}_{2} \mathrm{O}$ interface due to hydration by $\mathrm{D}_{2} \mathrm{O}$; (3) $\mathrm{H}-\mathrm{D}$ exchange of $\mathrm{NH}_{2}$ groups. This loss of interfacial contrast has been noted before in SANS studies of both hydrocarbon and fluorocarbon hydroxy surfactants $[33,44]$, therefore this is likely here considering the acrylamide groups bear labile amide protons. Hence, here SANS has only able to resolve an average contrast for the micellised surfactant against solvent and therefore an overall global fit has been conducted.

The self-assembled structure adopted by non-ionic Dynax ${ }^{\mathrm{TM}}$ DX2200 at all studied concentrations is best described by prolate spheroids, or globular micelles, with no observable structure factor over these concentrations and $Q$ ranges. This has been seen in other studies of non-ionic fluorinated surfactants $[33,45]$. From Table 3 it can be seen that on average the surfactant had an equatorial radius of $65 \AA$ and a polar radius of $28 \AA$. The calculated tip-to-toe length of this molecule is $\sim 63 \AA$, this therefore suggests that the molecule is fully extended at the extreme equatorial axis, but coiled at the extreme polar axis. This coiling has been previously observed using SANS in surfactants containing polyoxyethylene groups [46], therefore is likely to occur with the oligomeric acrylamide moieties.

The different aggregate structures can be explained by consideration of the surfactant packing parameter argument (Eq. (6)):

$P_{C}=v /\left(a_{0} l_{c}\right)$ 
where $\mathrm{v}$ is volume of the hydrophobic tail, $a_{0}$ is head group area and $l_{c}$ is the length of the hydrophobic chain. For each surfactant the tail volume and length effectively remains the same and the only factor changing is predominantly the head group area. As expected, the molecule with the largest headgroup (Dynax ${ }^{\mathrm{TM}}$ DX2200) formed ellipsoidal micelles, and the molecule with the smallest head group (Capstone $^{\mathrm{TM}}$ 1157) formed larger lamellar aggregates. Overall, three different self-assembled structures were observed for the three different fluorinated surfactants, highlighting how a change of surfactant headgroup can vastly affect self-assembly structure.

\section{Conclusions}

There are clear incentives to move away from the use of industrial fluorocarbon (FC) surfactants $[3,9-13,47]$. However, to practically achieve this, there must be an understanding of the important bulk and surface properties of these surfactants as both single and multi-component systems. Here, three typical industrial FC surfactants used in fire-fighting have been characterised by surface tension, fluorescence probe studies, neutron reflection and smallangle neutron scattering, so that links can be made between their structures and respective bulk and interfacial performance.

As expected, all surfactants have very low limiting surface tensions, with the lowest being observed for the zwitterionic surfactant $\left(\gamma_{C M C}=15.6 \mathrm{mN} \mathrm{m}^{-1}\right)$. Comparisons in limiting surface tension and critical micelle concentrations (CMC) between partially fluorinated surfactants in this study and fully fluorinated surfactants (sodium perfluorooctanoate (NaPFO) [28]) have been made (Tables 1 and S8), with clear differences being noted. It was interesting to note the differences in surface activity between the zwitterionic surfactant Capstone ${ }^{\mathrm{TM}} 1157$ and the anionic surfactant Dynax ${ }^{\mathrm{TM}}$ DX1030. Although they both have the same tail structure, but only differ in head group, the zwitterionic surfactant has a $\mathrm{CMC} \sim 6$ times lower and a $\gamma_{C M C} \sim 4 \mathrm{mN} \mathrm{m}^{-1}$ lower than the anionic surfactant. These results show how considerable changes in the interfacial properties of surfactants result from changes in surfactant chemical structure.

In depth analysis of the surface tension data using the Gibbs adsorption isotherm was prevented due to possible contamination owing to the commercial nature of these surfactants. In an attempt to circumvent this problem, neutron reflection was used to gain understanding of the surface films, by determining parameters such as surface excess and area per molecule. The range of surfactants used in this provided an easy to follow expected trend, the largest area per molecule $\left(\mathrm{A}_{c m c}\right)$ was observed in the largest molecule non-ionic Dynax ${ }^{\mathrm{TM}}$ DX2200, and smallest $\mathrm{A}_{c m c}$ in the smallest molecule zwitterionic Capstone ${ }^{\mathrm{TM}}$ 1157. Again it is interesting to see the differences when comparing the zwitterionic to the anionic surfactant. The charge on the anionic surfactant feeds through to an increase of $\sim 12 \AA^{2}$ in $\mathrm{A}_{c m c}$ and $\mathrm{a} \sim 1.2 \times 10^{-6} \mathrm{~mol} \mathrm{~m}^{-2}$ decrease in $\Gamma_{c m c}$. Another important observation was that the monolayer density of Capstone ${ }^{\mathrm{TM}} 1157$ appears to be comparable to that of a single fluorocarbon chain $\left(\sim 28 \AA^{2}\right)$ [37-39], suggesting that the molecules are reaching the limit at which they can physically fit at the air-water interface, further explaining why this molecule has such a low value of $\gamma_{c m c}\left(\sim 15.6 \mathrm{mN} \mathrm{m}^{-1}\right)$. Literature has been produced on NaPFO $[28,29,35]$ and although it was possible to make comparisons between NaPFO and anionic Dynax ${ }^{\mathrm{TM}}$ DX1030/ Capstone $^{\mathrm{TM}}$ 1157, it was instructive to compare results from these technical grade surfactants and literature standards. Dupont et al. [33] carried out experiments on a novel FC telomer surfactant, similar to Dynax ${ }^{\mathrm{TM}}$ DX2200 used in this study, which was used for comparison. For practical applications, it is important to have an understanding not only on the interfacial properties, but also bulk properties. An understanding of the bulk properties is important because links between self-assembly and rheology (viscoelasticity) can be made.

Through the use of small-angle neutron scattering (SANS) it has been possible to explore self-assembly structures. The differences observed in aggregation and self-assembly could be understood in terms of the packing parameter (Eq. (6)). Large head group

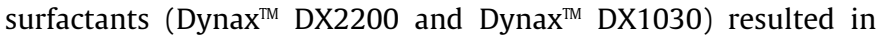
scattering consistent with spherical or ellipsoidal form factors, whereas the small head group surfactant (Capstone ${ }^{\mathrm{TM}}$ 1157) was better described by a lamellar-type form factor. Capstone ${ }^{\mathrm{TM}} 1157$ has been noted to be the only surfactant to display a noticeable increase of viscosity in solution, as previously reported by Kumar et al. [42] with similar FC zwitterionic surfactants. By having this understanding of how these surfactants self-assemble individually, more complex studies can be proposed to model systems closer to practical applications, i.e containing F/F Carbon or H/F Carbon surfactant mixtures.

This study has highlighted some of the important surfactant properties for fire-fighting applications, notably for aqueous filmforming foams (AFFFs). Surfactants with low surface tensions and CMCs are likely to perform better and provide a way of achieving the desired interfacial properties. As well as this, results have shown the sensitivity between the relationship of structure and performance between surfactants with similar tail groups but different head groups. This emphasises how changes in head group can feed through to a large difference in both bulk and surface behaviour. In addition, it has been shown how lab-based tensiometric techniques are not always reliable for analysis of technical grade FC surfactants. However, the utility of neutron reflection for systems and studies of this kind has been demonstrated. These results therefore provide important insight into structureperformance relationships in FC surfactants, and will point towards new ways to design more environmentally benign and effective FC surfactants in the future.

\section{Acknowledgements}

$\mathrm{CH}$ thanks Angus Fire Ltd. for the provision of a $\mathrm{PhD}$ studentship. The authors thank the UK Science and Technology Facilities Council (STFC) for allocation of beamtime at ISIS and ILL and associated grants for consumables and travel. This work benefited from SasView Small Angle Scattering Analysis Software Package, originally developed by the DANSE project under NSF award DMR-0520547.

\section{Appendix A. Supplementary material}

Supplementary data associated with this article can be found, in the online version, at https://doi.org/10.1016/j.jcis.2018.07.023.

\section{References}

[1] A. Ratzer, History and development of foam as a fire extinguishing medium, Ind. Eng. Chem. 48 (11) (1956) 2013-2016.

[2] C. Hill, J. Eastoe, Foams: from nature to industry, Adv. Colloid Interface Sci. 247 (2017) 496-513, https://doi.org/10.1016/j.cis.2017.05.013.

[3] C.A. Moody, J.A. Field, Perfluorinated surfactants and the environmental implications of their use in fire-fighting foams, Environ. Sci. Technol. 34 (18) (2000) 3864-3870

[4] A. Czajka, G. Hazell, J. Eastoe, Surfactants at the design limit, Langmuir 31 (30) (2015) 8205-8217.

[5] M.P. Krafft, J.G. Riess, Chemistry, physical chemistry, and uses of molecular fluorocarbon- hydrocarbon diblocks, triblocks, and related compounds unique apolar components for self-assembled colloid and interface engineering, Chem. Rev. 109 (5) (2009) 1714-1792.

[6] W.D. Harkins, A. Feldman, Films. the spreading of liquids and the spreading coefficient, J. Am. Chem. Soc. 44 (12) (1922) 2665-2685.

[7] K. Shinoda, T. Nomura, Miscibility of fluorocarbon and hydrocarbon surfactants in micelles and liquid mixtures. basic studies of oil repellent and fire extinguishing agents, J. Physi. Chem. 84 (4) (1980) 365-369. 
[8] B.Z. Dlugogorski, S. Phiyanalinmat, E.M. Kennedy, Dynamic surface and interfacial tension of AFFF and fluorine-free Class B foam solutions, Fire Saf. Sci. 8 (2005) 719-730.

[9] M. Filipovic, A. Woldegiorgis, K. Norström, M. Bibi, M. Lindberg, A.-H. Österås, Historical usage of aqueous film forming foam: a case study of the widespread distribution of perfluoroalkyl acids from a military airport to groundwater, lakes, soils and fish, Chemosphere 129 (2015) 39-45.

[10] W.J. Backe, T.C. Day, J.A. Field, Zwitterionic, cationic, and anionic fluorinated chemicals in aqueous film forming foam formulations and groundwater from US military bases by non-aqueous large-volume injection HPLC-MS/MS, Environ. Sci. Technol. 47 (10) (2013) 5226-5234.

[11] B.J. Place, J.A. Field, Identification of novel fluorochemicals in aqueous filmforming foams used by the US military, Environ. Sci. Technol. 46 (13) (2012) $7120-7127$.

[12] J. Giesy, K. Kannan, Perfluorochemical surfactants in the environment, Environ. Sci. Technol. 36 (7) (2002) 146A.

[13] J. Seow, C.W. Australia, Fire Fighting Foams with PerfluorochemicalsEnvironmental Review, 2013.

[14] K.M. Hinnant, M.W. Conroy, R. Ananth, Influence of fuel on foam degradation for fluorinated and fluorine-free foams, Colloids Surf., A 522 (2017) 1-17.

[15] L.A. D'Agostino, S.A. Mabury, Identification of novel fluorinated surfactants in aqueous film forming foams and commercial surfactant concentrates, Environ. Sci. Technol. 48 (1) (2013) 121-129.

[16] D. Korolchenko, S. Voevoda, Influence of spreading structure in an aqueous solution-hydrocarbon system on extinguishing of the flame of oil products, MATEC Web of Conferences, vol. 86, EDP Sciences, 2016, p. 04038.

[17] J.D. Hines, The preparation of surface chemically pure sodium n-dodecyl sulfate by foam fractionation, J. Colloid Interface Sci. 180 (2) (1996) 488-492.

[18] J. Eastoe, S. Nave, A. Downer, A. Paul, A. Rankin, K. Tribe, J. Penfold, Adsorption of ionic surfactants at the air - solution interface, Langmuir 16 (10) (2000) 4511-4518.

[19] J. Lu, E. Lee, R. Thomas, J. Penfold, S. Flitsch, Direct determination by neutron reflection of the structure of triethylene glycol monododecyl ether layers at the air/water interface, Langmuir 9 (5) (1993) 1352-1360.

[20] J. Hines, P. Garrett, G. Rennie, R. Thomas, J. Penfold, Structure of an adsorbed layer of n-dodecyl-N, N-dimethylamino acetate at the air/solution interface as determined by neutron reflection, J. Phys. Chem. B 101 (36) (1997) 7121-7126.

[21] H. Xu, P. Li, K. Ma, R.J. Welbourn, J. Penfold, D.W. Roberts, R.K. Thomas, J.T. Petkov, Adsorption of methyl ester sulfonate at the air-water interface: can limitations in the application of the gibbs equation be overcome by computer purification?, Langmuir 33 (38) (2017) 9944-9953

[22] Ö. Topel, B.A. Çakır, L. Budama, N. Hoda, Determination of critical micelle concentration of polybutadiene-block-poly (ethyleneoxide) diblock copolymer by fluorescence spectroscopy and dynamic light scattering, J. Mol. Liq. 177 (2013) 40-43.

[23] J. Webster, S. Holt, R. Dalgliesh, INTER the chemical interfaces reflectometer on Target Station 2 at ISIS, Phys. B: Phys. Condens. Matter (385-386) (2006) 1164-1166.

[24] A. Nelson, Co-refinement of multiple-contrast neutron/X-ray reflectivity data using MOTOFIT, J. Appl. Crystallogr. 39 (2) (2006) 273-276.

[25] J. Daillant, A. Gibaud, X-ray and Neutron Reflectivity: Principles and Applications, Vol. 770, Springer, 2008.

[26] J. Penfold, R. Thomas, The application of the specular reflection of neutrons to the study of surfaces and interfaces, J. Phys.: Condens. Matter 2 (6) (1990) 1369.

[27] Z. Derikvand, M. Riazi, Experimental investigation of a novel foam formulation to improve foam quality, J. Mol. Liq. 224 (2016) 1311-1318.

[28] E. Kissa, Fluorinated Surfactants and Repellents, Vol. 97, CRC Press, 2001.
[29] J. Lu, R. Ottewill, A. Rennie, Adsorption of ammonium perfluorooctanoate at the air-water interface, Colloids Surf., A 183 (2001) 15-26.

[30] S.K. Hait, S.P. Moulik, Determination of critical micelle concentration (CMC) of non-ionic surfactants by donor-acceptor interaction with lodine and correlation of CMC with hydrophile-lipophile balance and other parameters of the surfactants, J. Surfactants Deterg. 4 (3) (2001) 303-309.

[31] P.H. Elworthy, K.J. Mysels, The surface tension of sodium dodecylsulfate solutions and the phase separation model of micelle formation, J. Colloid Interface Sci. 21 (3) (1966) 331-347.

[32] K.J. Mysels, Surface tension of solutions of pure sodium dodecyl sulfate Langmuir 2 (4) (1986) 423-428.

[33] A. Dupont, J. Eastoe, P. Barthélémy, B. Pucci, R. Heenan, J. Penfold, D.C. Steytler, I. Grillo, Neutron reflection and small-angle neutron scattering studies of a fluorocarbon telomer surfactant, J. Colloid Interface Sci. 261 (1) (2003) $184-$ 190.

[34] S. An, J. Lu, R. Thomas, J. Penfold, Apparent anomalies in surface excesses determined from neutron reflection and the gibbs equation in anionic surfactants with particular reference to perfluorooctanoates at the air/water interface, Langmuir 12 (10) (1996) 2446-2453.

[35] E.A. Simister, E.M. Lee, J.R. Lu, R.K. Thomas, R.H. Ottewill, A.R. Rennie, J Penfold, Adsorption of ammonium perfluorooctanoate and ammonium decanoate at the air/solution interface, J. Chem. Soc., Faraday Trans. 88 (20) (1992) 3033-3041.

[36] E. Lee, R. Thomas, J. Penfold, R. Ward, Structure of aqueous decyltrimethylammonium bromide solutions at the air water interface studied by the specular reflection of neutrons, J. Phys. Chem. 93 (1) (1989) 381-388.

[37] J. Eastoe, A. Paul, A. Rankin, R. Wat, J. Penfold, J.R. Webster, Fluorinated nonionic surfactants bearing either $\mathrm{CF}_{3}$ or $\mathrm{H}-\mathrm{CF}_{2}$ - terminal groups: Adsorption at the surface of aqueous solutions, Langmuir 17 (25) (2001) 7873-7878.

[38] A. Downer, J. Eastoe, A.R. Pitt, J. Penfold, R.K. Heenan, Adsorption and micellisation of partially-and fully-fluorinated surfactants, Colloids Surf., A $156(1-3)(1999)$ 33-48.

[39] C. Arrington Jr., G. Patterson, Colloidal properties of highly fluorinated alkanoic acids, J. Phys. Chem. 57 (2) (1953) 247-250.

[40] E.G.R. Putra, A. Ikram, Nanosize structure of self-assembly sodium dodecy sulfate: A study by small-angle neutron scattering (SANS), Indonesian J. Chem. 6 (2) (2010) 117-120.

[41] S.S. Berr, R.R. Jones, Small-angle neutron scattering from aqueous solutions of sodium perfluorooctanoate above the critical micelle concentration, J. Phys. Chem. 93 (6) (1989) 2555-2558.

[42] R. Kumar, G.C. Kalur, L. Ziserman, D. Danino, S.R. Raghavan, Wormlike micelles of a C22-tailed zwitterionic betaine surfactant: from viscoelastic solutions to elastic gels, Langmuir 23 (26) (2007) 12849-12856.

[43] D.S. Sivia, Elementary Scattering Theory: for X-ray and Neutron Users, Oxford University Press, 2011.

[44] J. Eastoe, P. Rogueda, A.M. Howe, A.R. Pitt, R.K. Heenan, Properties of new glucamide surfactants, Langmuir 12 (11) (1996) 2701-2705.

[45] L.K. Shrestha, S.C. Sharma, T. Sato, O. Glatter, K. Aramaki, Small-angle X-ray scattering (SAXS) study on non-ionic fluorinated micelles in aqueous system, J. Colloid Interface Sci. 316 (2) (2007) 815-824.

[46] P.A. FitzGerald, T.W. Davey, G.G. Warr, Micellar structure in gemini non-ionic surfactants from small-angle neutron scattering, Langmuir 21 (16) (2005) $7121-7128$.

[47] A.V. Vinogradov, D. Kuprin, I. Abduragimov, G. Kuprin, E. Serebriyakov, V.V. Vinogradov, Silica foams for fire prevention and fire-fighting, ACS Appl. Mater. Interfaces 8 (1) (2016) 294-301. 